\title{
The expression of ATP-binding cassette transporters in hypertensive patients
}

\author{
Mingzhi Xu, Huali Zhou, Qing Gu and Chengjiang Li
}

Cholesterol efflux is regulated by cholesterol transporters, including adenosine triphosphate-binding cassette transporters, A1, G1 (ABCA1, ABCG1), and scavenger receptor class B type I (SR-BI). We have investigated whether the expression of these transporters/receptor is altered in patients with hypertension and also studied their functional effects in cholesterol efflux. The newly diagnosed hypertensive patients, as well as age- and gender-matched healthy controls were recruited. mRNA of $A B C A 1$, $A B C G 1$ and $S R-B I$ in monocytes was measured. The functional effects of the three transporters/receptor and cholesterol efflux from monocyte-derived macrophages ex vivo were also determined. The expression of $A B C A 1$ and $A B C G 1$ was significantly decreased in the newly diagnosed untreated hypertensive patients compared with that in healthy controls. The levels of $A B C A 1$ and $A B C G 1$ were negatively associated with blood pressure, and the reduction of $A B C A 1$ and $A B C G 1$ could be reversed by antihypertensive therapy. No significant associations between plasma lipids, oxidized low-density lipoprotein (LDL) and the expression of $A B C A 1$ or $A B C G 1$ were found. Cholesterol efflux from monocyte-derived macrophages to autologous serum, apolipoprotein $\mathrm{Al}$ (apoAI) or high-density lipoprotein (HDL) was impaired in hypertensive patients. Cholesterol efflux to autologous serum or apoAI was associated with the expression of $A B C A 1$, whereas cholesterol efflux to autologous serum or HDL was associated with the expression of $A B C G 1$. The expression of $A B C A 1$ and $A B C G 1$ in monocytes is reduced in hypertensive patients, which could be reversed by anti-hypertensive therapy. The reduction in $A B C A 1 / A B C G 1$ is associated with the impairment of cholesterol efflux from monocyte-derived macrophages.

Hypertension Research (2009) 32, 455-461; doi:10.1038/hr.2009.46; published online 24 April 2009

Keywords: ATP-binding cassette transporters; cholesterol efflux; reverse cholesterol transport; scavenger receptor class B type I

\section{INTRODUCTION}

Reverse cholesterol transport (RCT) is a pathway that transports cholesterol from extrahepatic cells and tissues to the liver for excretion, and cholesterol efflux represents the first critical step of RCT. ${ }^{1}$ Specifically, cholesterol efflux from macrophages represents only a small fraction of the overall cellular cholesterol efflux, but it is probably the most important with regard to atherosclerosis, and it has recently been termed macrophage RCT, as excess lipid accumulation within macrophages in the peripheral artery wall may lead to foam cell formation. ${ }^{1}$

Cholesterol efflux is a complex process that involves at least two aspects, namely extracellular cholesterol acceptors, mainly referring to high-density lipoprotein (HDL) and cellular cholesterol transporters/ receptors. Macrophage adenosine triphosphate (ATP)-binding cassette transporters A1, G1 (ABCA1, ABCG1) and scavenger receptor class B type I (SR-BI) are the three best-characterized cellular transporters/ receptor that are involved in determining the macrophage cholesterol efflux in vitro and in animal models. ${ }^{2,3}$ ABCA1 mediates cholesterol efflux from macrophages to lipid-free/poor apolipoprotein AI (apoAI), whereas ABCG1 mediates the macrophage cholesterol efflux to phospholipids containing HDL particles in vitro. SR-BI is expressed in hepatocytes and macrophages and also mediates cholesterol efflux to mature HDL in vitro.

Hypertension is associated with an increased risk of atherosclerosis and cardiovascular diseases (CVDs). However, few studies on cholesterol efflux and RCT in hypertensive conditions have been carried out. It has been well established that the HDL cholesterol level is strongly inversely associated with CVD. ${ }^{4,5}$ Not only the HDL level, but also HDL compositions may account for the protective activity of HDL. ${ }^{6}$ It has been reported that the HDL concentration of patients with hypertension does not differ from that of healthy controls, but the phospholipid compositions of HDL are altered, which may impair the functional capacity of HDL in inducing cholesterol efflux. ${ }^{7}$

On the cellular aspects of cholesterol efflux, no studies regarding the expression of the three important transporters/receptor of $A B C A 1$, $A B C G 1$ and $S R-B I$, and their functional effects of cholesterol efflux in hypertensive conditions, have been carried out. Therefore, the aims of this study were to investigate whether the expression of $A B C A 1$, $A B C G 1$ and $S R-B I$ in peripheral blood monocytes was changed in hypertensive patients, as well as its alteration on anti-hypertensive 
therapy, and to determine whether these alterations might affect cholesterol efflux from monocyte-derived macrophages ex vivo.

\section{METHODS}

The study was approved by the Ethics Committee of the College of Medicine, Zhejiang University, China, and informed consent was obtained from each participant. We certify that all applicable institutional and governmental regulations regarding the ethical use of human volunteers were followed-up during this research. Hypertensive patients and healthy controls were recruited to compare the difference in the expression of ABCA1, ABCG1 and SR-BI. We also followed-up the alteration of these transporters/receptor after controlling their blood pressure compared with that before anti-hypertensive therapy in our hypertensive patients. Specifically, 20 newly diagnosed hypertensive patients and 20 age- and gender-matched healthy controls were recruited from the community during their annual examination. All patients were confirmed to have no other known diseases, including diabetes, obesity, CVD, dyslipidemia, thyroid diseases, renal diseases, chronic respiratory diseases or any other acute and chronic disease conditions, or any current infectious condition. Primary hypertension was diagnosed according to the following criteria: (1) age $>45$ years; (2) the family history of hypertension is positive; (3) systolic pressure $\geqslant 150 \mathrm{~mm} \mathrm{Hg}$, and/or diastolic pressure $\geqslant 95 \mathrm{~mm} \mathrm{Hg}$, which was measured at resting condition and confirmed thrice on different days; (4) none of the patients had ever used anti-hypertensive drugs. The patients were subjected to anti-hypertensive therapy, including diet, physical activities and Nifedipine Sustained-release Capsules (Yangtze River Pharmaceutical Group, Jiangsu, China) for 3 months. We chose nifedipine because it was relatively affordable for the general Chinese population and its anti-hypertensive effects were fairly good for newly diagnosed patients with hypertension. Most importantly, it has been reported that there is no direct effect of nifedipine in modulating the expression of $\mathrm{ABC}$ transporters, ${ }^{8}$ which might help to exclude potential confounding factors to observe the relationship between $\mathrm{ABC}$ transporters and blood pressure. The dosage of nifedipine was 20-60 mg daily according to the blood pressure level individually.

Age, gender, weight, height, waist, blood pressure, history of smoking and medications were recorded. Body mass index (BMI) was calculated as weight (kg) per squared height $\left(\mathrm{m}^{2}\right)$. The mean blood pressure was calculated as diastolic pressure $+1 / 3 \times($ systolic pressure-diastolic pressure). An adjustment of the potential confounding factors was made in comparison of mean difference or multiple analyses. Blood samples were taken after an overnight fast. The samples were centrifuged, and plasma and serum levels were aliquoted and immediately frozen at $-80^{\circ} \mathrm{C}$. Plasma levels of total cholesterol, triglyceride, HDL cholesterol and low-density lipoprotein (LDL) cholesterol were obtained from clinical records. Plasma-oxidized LDL was measured using the oxidised LDL ELISA kit (Mercodia, Winston Salem, NC, USA). All the measurements were made before and after anti-hypertensive therapy.

Peripheral blood monocytes were isolated as described earlier with slight modifications. ${ }^{9}$ Briefly, $40 \mathrm{ml}$ of fasting blood sample was collected from each patient using $4 \mathrm{ml}$ of $3.8 \%$ of sodium citrate as the anti-coagulant. Blood monocytes were isolated using Ficoll-Paque solution (Amersham Biosciences, Piscataway, NJ, USA) according to the manufacturer's instructions. Briefly, the blood sample was layered carefully on the Ficoll-Paque solution and thereafter centrifuged at $400 \mathrm{~g}$ for $30 \mathrm{~min}$ at $18-20^{\circ} \mathrm{C}$. Mononuclear cells at the interface were collected, washed thrice with sterile phosphate-buffered saline (PBS) and then re-suspended with RPMI 1640 culture medium (Gibco, Grand Island, NY, USA). Mononuclear cells in the medium were added to culture plates and incubated in a cell culture incubator for $2-3 \mathrm{~h}$. Unattached lymphocytes were removed gently. The purity of the attached monocytes was initially assessed morphologically under light microscopy, and then by non-specific esterase staining and sodium fluoride inhibition test (positive rate $>85 \%$ ), and cell viability was measured by the trypan blue exclusion test (viable rate $>90 \%$ ). The specificity and sensitivity of this method in determining monocyte purity is comparable with the method of fluorescence activated cell sorting. ${ }^{10}$ Monocytes were collected for cholesterol efflux assay immediately or stored at $-70{ }^{\circ} \mathrm{C}$ for RNA extraction and for real-time quantitative PCR.

Cholesterol efflux from monocyte-derived macrophages was evaluated as the functional effects of the three cellular transporters/receptor as described earlier, with slight modifications. ${ }^{9}$ The attached monocytes were differentiated into macrophages ex vivo by incubating in the RPMI 1640 medium containing $10 \%$ autologous serum for 6 days according to Gianturco et al. ${ }^{11}$ Thereafter, cellular cholesterol was labeled with tracer $[3 \mathrm{H}]$ cholesterol (Amersham Biosciences) for $24 \mathrm{hrs}$, and the final concentration of the tracer was $1 \mu \mathrm{Ci}, 0.01 \mu \mathrm{g} /$ well, $10^{6}$ mononuclear cells. Autologous serum was diluted to $5 \%$ with culture medium and used to induce cholesterol efflux from the labeled cells for $4 \mathrm{~h}$. Cholesterol efflux, expressed in percentage, was calculated as the count in the medium divided by the total count of medium and cells in each well. Each sample was determined in triplicate. A similar assay of cholesterol efflux was repeated when using apoAI (Biodesign, Saco, ME, USA) $20 \mu \mathrm{g} \mathrm{ml}^{-1}$, or HDL (isolated from a pool of normal plasma by ultra-centrifugation) $50 \mu \mathrm{g} \mathrm{ml}^{-1}$ as a cholesterol acceptor instead of $5 \%$ autologous serum. The levels of cholesterol efflux to various cholesterol acceptors were subtracted by the basal level of cholesterol efflux (no additional cholesterol acceptor was contained in the medium). Monocyte isolation and cholesterol efflux assay were assayed by the same experimenter to minimize the variation and five samples could be handled within 1 day at the maximum. A volume of $20-\mathrm{ml}$ blood was collected from the experimenter herself as a reference sample, and cholesterol efflux from her macrophages to autologous serum, apoAI or HDL was measured side-by-side on each day. Cholesterol efflux carried out on different days was normalized to the reference level, which was always included on each day. The interassay coefficient of variation was $6.1 \%$.

Total RNA was extracted using Total RNA Extraction Miniprep System (Viogene, Sunnyvale, CA, USA) according to the protocol provided by the manufacturer and quantified spectrophotometrically. cDNA was synthesized using TaqMan reagents (Applied Biosystems, Framingham, MA, USA) according to the manufacturer's instructions. The mRNA level was determined according to Zhou et al. ${ }^{9}$ with slight modifications. Briefly, commercial primer/probe sets for target genes ABCA1 (Hs00194045_m1), ABCG1 (Hs00245154_m1), SR-BI (Hs00194092_m1) and endogenous reference gene, GAPDH, were used. PCR was performed in 96-well plates using $50 \mathrm{ng}$ cDNA mixed with primers, probes and Taqman Universal PCR Master Mix (Applied Biosystems) in a total volume of $50 \mu \mathrm{l}$ in an ABI PRISM 7700 sequence detector (Applied Biosystems Inc., Austin, TX, USA). All cDNA samples were assayed in duplicate. Each target gene and GAPDH was assayed on the same samples in separate tubes. This allowed standardization of the amount of target gene to the internal reference gene to control for different amounts of cDNA used. The standardized target gene was then compared with a reference sample and expressed as folds of the reference sample.

Numerical data were expressed as mean and s.d. Data that were not normally distributed were logarithmically transformed before analyses were performed. Mean differences of continuous variables were evaluated using Student's $t$-test between two independent groups of healthy controls and untreated hypertensive patients, or using a paired $t$-test between two dependent groups before and after anti-hypertensive therapy. The $\chi^{2}$-test was used to compare differences in the proportions between groups. Pearson's correlations were used to test the relationship between two variables, and the linear regression model was used to assess the relationships between multiple variables simultaneously. $P$-value $<0.05$ was considered as statistically significant.

\section{RESULTS}

The general characteristics and lipid profile of the patients are shown in Table 1. Age and gender were matched between the two groups. The proportion of smokers and BMI was not significantly different between the two groups. As expected, both systolic pressure and diastolic pressure were significantly higher in hypertensive patients compared with that in healthy controls. Plasma levels of total cholesterol, HDL, LDL and triglycerides were comparable between healthy controls and hypertensive patients. Circulating oxidized LDL was significantly increased in untreated hypertensive patients compared with that in healthy controls. Oxidized LDL tended to be decreased after anti-hypertensive therapy, but it did not reach statistical significance. 
Table 1 Clinical characteristics and lipid profile of healthy controls and hypertensive patients before or after anti-hypertensive therapy

\begin{tabular}{|c|c|c|c|}
\hline & \multirow[b]{2}{*}{ Healthy controls } & \multicolumn{2}{|c|}{ Hypertensive patients } \\
\hline & & Before therapy & After therapy \\
\hline Age (years) & $50.3 \pm 6.9$ & \multicolumn{2}{|c|}{$51.2 \pm 6.3$} \\
\hline Gender (male/female, \%) & $40 / 60$ & \multicolumn{2}{|c|}{$45 / 55$} \\
\hline Smokers (\%) & 10 & \multicolumn{2}{|c|}{15} \\
\hline $\mathrm{BMI}\left(\mathrm{kg} \mathrm{m}^{-2}\right)$ & $21.5 \pm 1.9$ & $22.7 \pm 3.1$ & $21.8 \pm 2.1$ \\
\hline Systolic pressure (mm Hg) & $119.1 \pm 12.9$ & $165.2 \pm 14.1^{\ddagger}$ & $128.2 \pm 15.1^{\#}$ \\
\hline Diastolic pressure $(\mathrm{mm} \mathrm{Hg})$ & $72.1 \pm 6.9$ & $98.9 \pm 7.9 \ddagger$ & $75.8 \pm 6.6^{\#}$ \\
\hline Total cholesterol $\left(\mathrm{mmol} \mathrm{I}^{-1}\right)$ & $5.08 \pm 0.93$ & $5.22 \pm 0.98$ & $5.16 \pm 0.92$ \\
\hline LDL cholesterol $\left(\mathrm{mmoll}^{-1}\right)$ & $2.93 \pm 0.77$ & $3.09 \pm 0.77$ & $3.03 \pm 0.86$ \\
\hline HDL cholesterol $\left(\mathrm{mmoll}^{-1}\right)$ & $1.50 \pm 0.34$ & $1.41 \pm 0.36$ & $1.48 \pm 0.39$ \\
\hline Triglyceride $\left(\mathrm{mmol} \mathrm{I}^{-1}\right)$ & $0.99(0.68)$ & $1.16(0.97)$ & $1.09(0.91)$ \\
\hline Oxidized LDL (mU I-1) & $9.6 \pm 1.3$ & $13.1 \pm 1.6^{*}$ & $10.4 \pm 1.5$ \\
\hline
\end{tabular}

Abbreviations: BMI, body mass index; HDL, high-density lipoprotein, LDL, low-density lipoprotein

Data were expressed as mean $\pm \mathrm{s}$.d. or median (interquartile range).

${ }^{\star} P<0.05,{ }^{\ddagger} P<0.001$ vs. healthy controls using Student's $t$-test, ${ }^{\#} P<0.001$ vs. before therapy using a paired $t$-test.

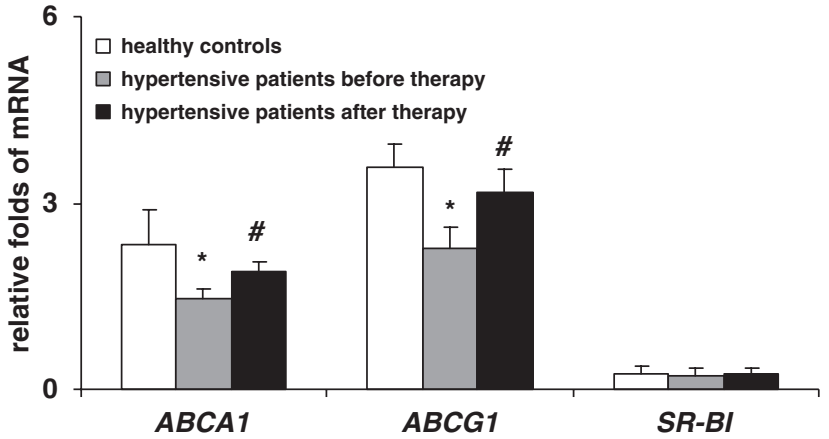

Figure 1 mRNA expression of $A B C A 1, A B C G 1$ and $S R-B I$ in fresh blood monocytes isolated from healthy controls and hypertensive patients before and after anti-hypertensive therapy. Data were expressed as mean \pm s.d. ${ }^{*} P<0.05$ compared with healthy controls, ${ }^{*} P<0.05$ compared with hypertensive patients before therapy. $A B C A 1$, adenosine triphosphatebinding cassette transporter $A 1 ; A B C G 1$, adenosine triphosphate-binding cassette transporter G1; SR-BI, scavenger receptor class B type I.

Peripheral blood monocytes mainly expressed $A B C A 1$ and $A B C G 1$, and the expression of $S R-B I$ was relatively low (Figure 1 ). There was a significant reduction in the mRNA levels of $A B C A 1$ and $A B C G 1$ in untreated hypertensive patients compared with that in healthy controls (Figure 1), which remained significant after adjusting for age, gender and smoking status. In contrast, the level of SR-BI was comparable between the two groups (Figure 1).

On correlation analysis, mRNA levels of $A B C A 1$ and $A B C G 1$ were correlated with mean blood pressure in all the subjects of healthy controls and untreated hypertensive patients (Figure 2). We have also analyzed the correlations within each separate group, and found similar associations between the expression of $A B C A 1 / A B C G 1$ and blood pressure (Table 2). However, some of them did not reach statistical significance, which might be because of the relatively small sample size. No significant association between the expression of $S R-B I$ and blood pressure was found. We also did not find any significant associations between plasma lipids, oxidized LDL and the
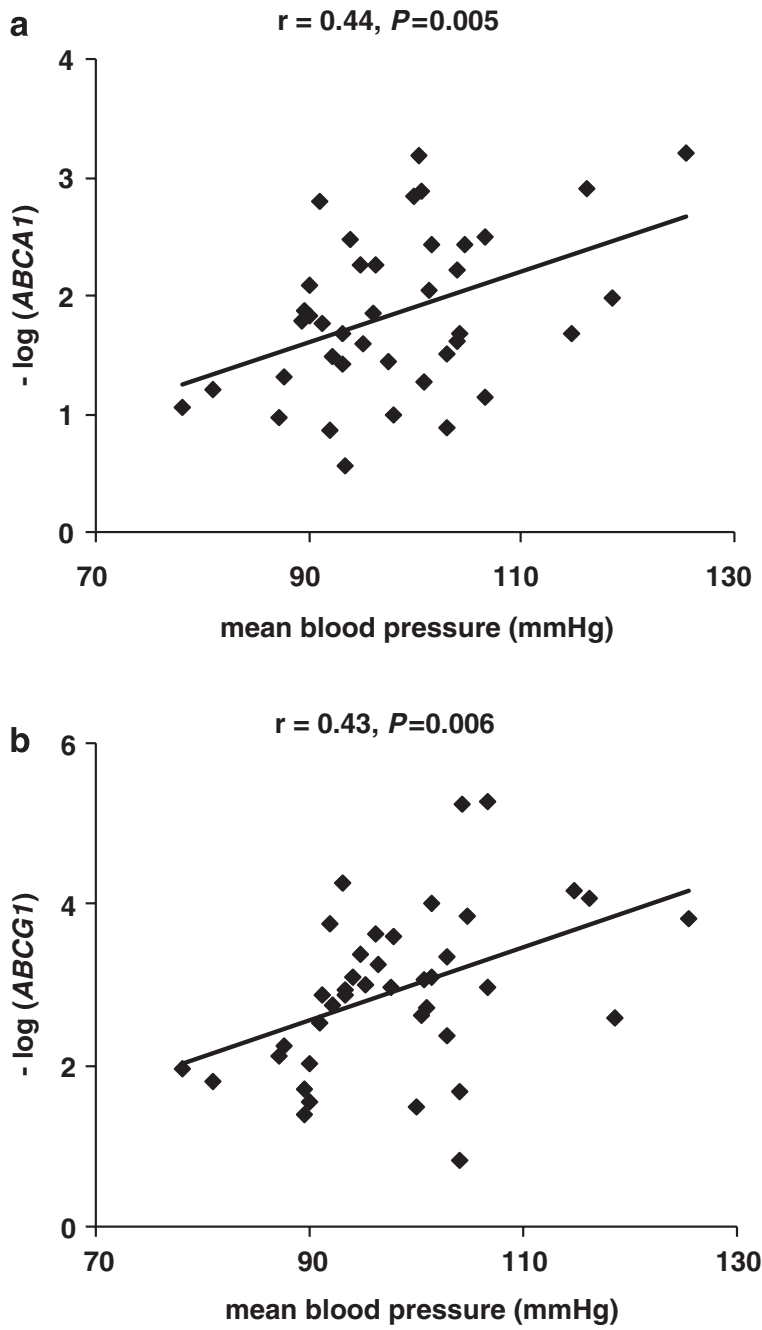

Figure 2 The associations between mean blood pressure and the mRNA expression of $A B C A 1$ (a), $A B C G 1$ (b) in monocytes in all the participants combining healthy controls and untreated hypertensive patients. The level of $A B C A 1$ and $A B C G 1$ was negatively transformed logarithmically before analysis. $A B C A 1$, adenosine triphosphate-binding cassette transporter $\mathrm{A} 1$; $A B C G 1$, adenosine triphosphate-binding cassette transporter G1.

expression of $A B C A 1, A B C G 1$ and SR-BI (Table 2). Further multiple regression analysis showed that mean blood pressure was an independent determinant of $A B C A 1 / A B C G 1$ mRNA expression, after adjusting potential confounding factors, such as age, gender, BMI, smoking, alcohol drinking and the mean daily dosage of nifedipine (Tables 3 and 4).

The above correlation and multiple regression analysis suggested that the reduction of the $A B C A 1 / A B C G 1$ expression in hypertensive patients might be, at least partially, because of high blood pressure. To confirm this, we also measured the expression of $A B C A 1$ and $A B C G 1$ in hypertensive patients after anti-hypertensive therapy. The levels of $A B C A 1$ and $A B C G 1$ were increased after blood pressure was controlled by nifedipine (Figure 1). It was noted that the alteration of $A B C A 1 /$ $A B C G 1$ expression was not related to the mean daily dosage of nifedipine, which was consistent with an earlier report that nifedipine may not directly modulate the expression of $\mathrm{ABC}$ transporters. ${ }^{8}$

To determine whether the reduction of the $A B C A 1 / A B C G 1$ expression in hypertensive patients might have a functional effect, cholesterol efflux from monocyte-derived macrophages to various 
Table 2 Correlations between the expression of $A B C A 1, A B C G 1, S R-B I$, and plasma lipids, oxidized LDL and mean blood pressure in each separate group

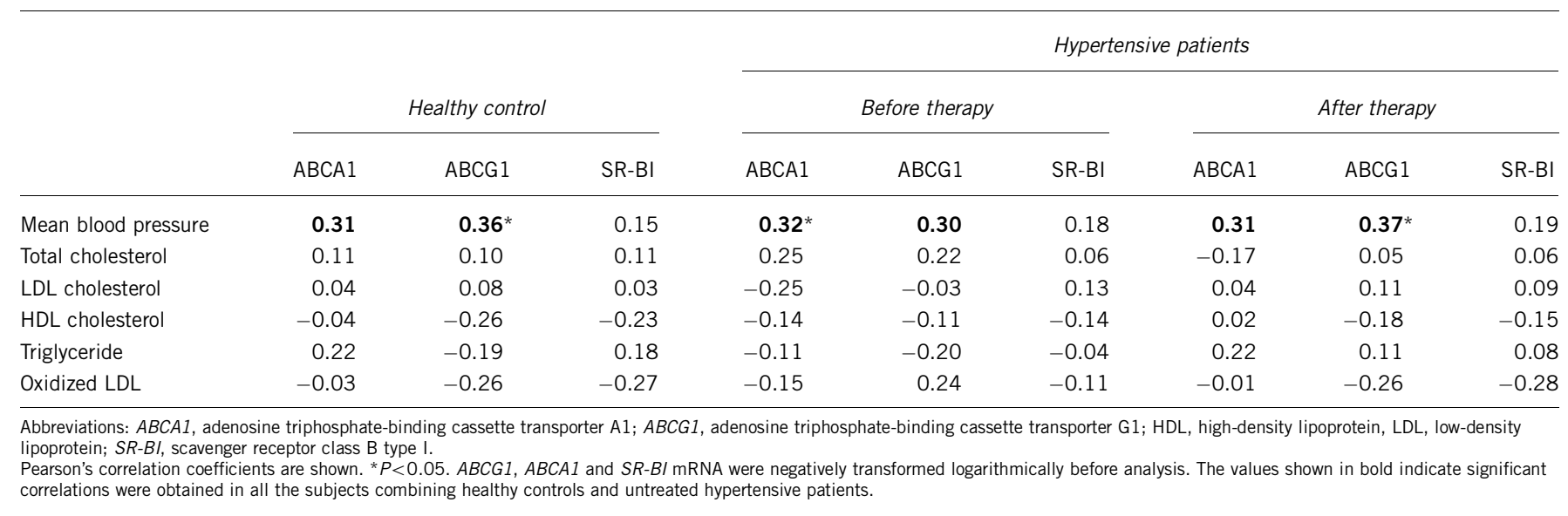

Table 3 General linear model univariate analysis with $-\log (A B C A 1)$ as the dependent variable in all the subjects of healthy controls and untreated hypertensive patients

\begin{tabular}{|c|c|c|c|c|}
\hline Intercept & 1.189 & 1.427 & & 0.411 \\
\hline Mean blood pressure & 0.032 & 0.011 & 0.460 & 0.006 \\
\hline Gender & -0.115 & 0.235 & -0.084 & 0.626 \\
\hline Body mass index & 0.312 & 0.140 & 0.346 & 0.040 \\
\hline Smoking & 0.163 & 0.243 & 0.129 & 0.506 \\
\hline
\end{tabular}

Abbreviation: ABCA1, adenosine triphosphate-binding cassette transporter A1.

$R^{2}$ of the model $=33.3 \%$. $A B C A 1$ mRNA expression was negatively transformed logarithmically before analysis. Gender was entered as a categorical variable (male $=1$, female $=2$ ), smoking (no=0, yes $=1$ ), alcohol drinking ( $\mathrm{no}=0$, yes $=1$ ). Those factors shown in bold reached statistical significance.

Table 4 General linear model univariate analysis with $-\log (A B C G 1)$ as the dependent variable in all the subjects of healthy controls and untreated hypertensive patients

\begin{tabular}{|c|c|c|c|c|}
\hline Intercept & 1.509 & 2.253 & & 0.508 \\
\hline Mean blood pressure & 0.046 & 0.017 & 0.444 & 0.012 \\
\hline Gender & -0.203 & 0.371 & -0.099 & 0.587 \\
\hline Body mass index & 0.240 & 0.221 & 0.187 & 0.286 \\
\hline Smoking & 0.269 & 0.383 & 0.143 & 0.489 \\
\hline
\end{tabular}

Abbreviation: $A B C A 1$, adenosine triphosphate-binding cassette transporter $\mathrm{A} 1$.

$R^{2}$ of the model $=24.8 \%$. ABCG1 mRNA expression was negatively transformed logarithmically before analysis. Gender was entered as a categorical variable (male $=1$, female $=2$ ), smoking (no=0, yes $=1$ ), alcohol drinking $(\mathrm{no}=0$, yes $=1$ ). Those factors shown in bold reached statistical significance.

cholesterol acceptors was measured. Cholesterol efflux from monocyte-derived macrophages to autologous serum, apoAI or HDL was reduced in hypertensive patients compared with that in healthy controls. Cholesterol efflux from monocyte-derived macrophages to autologous serum, apoAI or HDL in hypertensive patients was increased after anti-hypertensive therapy compared with that before therapy (Figure 3).
Furthermore, cholesterol efflux to autologous serum was associated with the expression of $A B C A 1$ and $A B C G 1$, but not with that of $S R-B I$ (Table 5). As ABCA1 mainly mediates cholesterol efflux to lipid-free apoAI, and $A B C G 1$ mainly mediates cholesterol efflux to mature HDL, we measured cholesterol efflux from monocyte-derived macrophages using apoAI or HDL as cholesterol acceptors instead. As expected, cholesterol efflux to apoAI was associated with the 


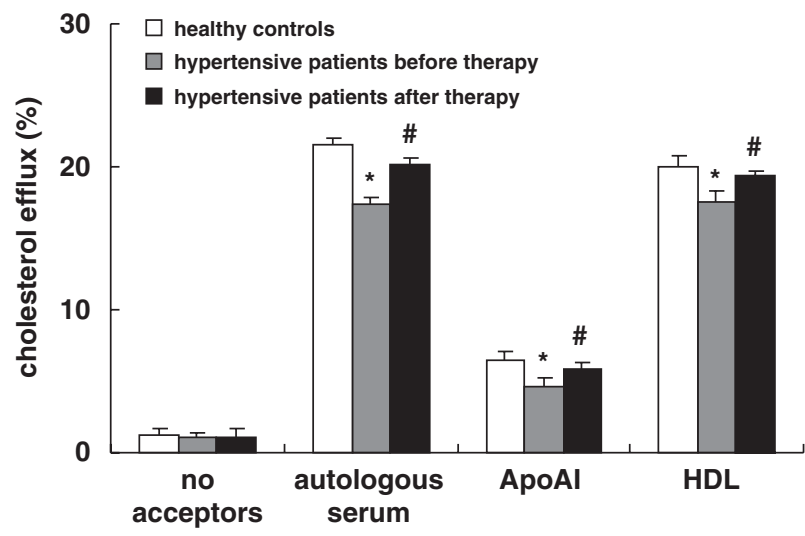

Figure 3 Cholesterol efflux from monocyte-derived macrophages to $5 \%$ autologous serum, apoAl $\left(20 \mu \mathrm{g} \mathrm{ml}^{-1}\right)$, or $\mathrm{HDL}\left(50 \mu \mathrm{g} \mathrm{m} \mathrm{I}^{-1}\right)$ in the respective group of healthy controls, hypertensive patients before and after therapy. Cholesterol efflux to various cholesterol acceptors had been subtracted by the basal level of cholesterol efflux, which was referred to as cholesterol efflux when no additional cholesterol acceptors were contained in the medium. Data were expressed as mean \pm s.d. ${ }^{*} P<0.05$ compared with healthy controls, $\# P<0.05$ compared with the untreated hypertensive patients. apoAI, apolipoprotein Al; HDL, high-density lipoprotein.

Table 5 Correlations between the expression of $A B C A 1, A B C G 1$, $S R-B I$ and cholesterol efflux from monocyte-derived macrophages to various acceptors in all the subjects combining healthy controls and untreated hypertensive patients

\begin{tabular}{|c|c|c|c|}
\hline & \multicolumn{3}{|c|}{ Cholesterol efflux to various acceptors } \\
\hline & Autologous serum & apoAl & $H D L$ \\
\hline$A B C A 1$ & $0.36^{*}$ & $0.38^{*}$ & 0.22 \\
\hline ABCG1 & $0.39 *$ & 0.18 & $0.42 *$ \\
\hline$S R-B I$ & 0.14 & 0.11 & 0.21 \\
\hline
\end{tabular}

Abbreviations: ABCA1, adenosine triphosphate-binding cassette transporter A1; ABCG1, adenosine triphosphate-binding cassette transporter $\mathrm{Gl}$; apoAl, apolipoprotein $\mathrm{Al}$; SR-BI, scavenger receptor class B type I.

Pearson's correlation coefficients are shown. ${ }^{*} P<0.05$. ABCG1, ABCA1 and $S R-B I$ mRNA were transformed logarithmically before analysis.

expression of $A B C A 1$, but not with that of $A B C G 1$ and $S R-B I$ (Table 5). Cholesterol efflux to HDL was associated with the expression of $A B C G 1$, but not with that of $A B C A 1$ and SR-BI (Table 5). These findings suggest that the reduction of $A B C A 1$ might lead to the impairment of cholesterol efflux from monocyte-derived macrophages to apoAI, and the reduction of $A B C G 1$ might lead to the impairment of cholesterol efflux from monocyte-derived macrophages to HDL. As a result, the reduction of $A B C A 1 / A B C G 1$ might lead to the impairment of cholesterol efflux from monocyte-derived macrophages to autologous serum ex vivo, which mimic an early step of macrophage RCT.

\section{DISCUSSION}

Cellular cholesterol efflux, being the first step of RCT, plays an important role in reducing the accumulation of lipids in the arterial wall and in preventing the development of atherosclerosis. It has recently been shown that cholesterol efflux from mouse macrophages in vivo is mainly mediated by ABCA1 and ABCG1, but not by SR-BI. ${ }^{12}$ This is the first study to examine the expression of these three major cholesterol transporters/receptor in human hypertensive patients. We have found that among the three cellular cholesterol transporters/ receptor in our human patients, $A B C G 1$ expression was the most abundant, followed by $A B C A 1$, whereas the level of $S R-B I$ expression was relatively low, which is consistent with the earlier finding by Zhou et al. ${ }^{9}$ We have clearly shown that the expression of $A B C A 1$ and $A B C G 1$ was significantly reduced in the newly diagnosed hypertensive patients, whereas the level of $S R-B I$ was similar to that in healthy controls. Furthermore, we found that blood pressure level was negatively associated with the expression of $A B C A 1$ and $A B C G 1$, and anti-hypertensive therapy could reverse the reduction of $A B C A 1$ and $A B C G 1$ levels in these hypertensive patients. In this study, we had compared the expression of $\mathrm{ABC}$ transporters between healthy controls and untreated hypertensive patients, as well as that in hypertensive patients between before and after anti-hypertensive therapy. If we had also followed-up those healthy controls for the second visit, more information about whether anti-hypertensive therapy could reverse $A B C A 1 / A B C G 1$ to a normal level could be obtained. This is a limitation of our experimental design and should be improved in our future work.

It is noted that nifedipine itself might not directly modulate the expression of $\mathrm{ABC}$ transporters, as we found that the alteration of $A B C A 1 / A B C G 1$ expression was not related to the mean daily dosage of nifedipine, which was consistent with an earlier report by Hasegawa et al. ${ }^{8}$ These findings suggested that the reduction of the ABCA1/ $A B C G 1$ expression in our patients might be because of their hypertensive condition. The regulation of macrophage $A B C A 1$ and $A B C G 1$ expression is responsive to cell cholesterol status, and cholesteroldependent transcription is mediated through the activation of peroxisome proliferator-activated receptor- $\gamma(\operatorname{PPAR} \gamma)$ and liver X receptor- $\alpha$ $(\operatorname{LXR} \alpha) .{ }^{13,14}$ It has been reported that PPAR $\gamma$ expression is decreased in the lung tissue from patients with pulmonary hypertension, and high fluid shear stress can decrease its expression in culture cells in vitro. ${ }^{15}$ As it has been widely known, PPAR $\gamma$ agonists (known as thiazolidinediones) can significantly reduce subsequent cardiovascular event rates in hypertensive patients. ${ }^{16-18}$ Our data give a clue that one of the underlying mechanisms through which PPAR $\gamma$ agonists show their cardiovascular actions might be through the activation of $\operatorname{PPAR} \gamma: \operatorname{LXR} \alpha$ and the subsequent induction of their target genes of ABCA1/ABCG1.

We also found that circulating oxidized LDL, an endogenous LXR agonist, increased in hypertensive patients, and that oxidized LDL was not correlated with the levels of $A B C A 1 / A B C G 1$. These findings suggested that the expression of $A B C A 1 / A B C G 1$ in our hypertensive patients might also be regulated through the LXR-independent pathway. It has been reported that reactive oxygen species (ROS) and advanced glycation end products (AGEs) might be involved in the regulation of $\mathrm{ABC}$ transporters through LXR-independent mechanisms. ${ }^{19,20}$ In both animal models and in humans, increased blood pressure has been associated with oxidative stress and the generation of ROS; ${ }^{21}$ ROS is involved in the downregulation of $A B C A 1$ expression in macrophages, ${ }^{19}$ which might be a potential underlying mechanism, whereby the expression of $A B C A 1$ decreased in hypertensive conditions. AGEs are also associated with hypertension, ${ }^{22}$ and may reduce the expression of $A B C G 1$ in vitro, ${ }^{20}$ as well as in type II diabetic patients. ${ }^{9}$ Further investigation of the association between the reduction of $A B C A 1 / A B C G 1$ in hypertensive patients and the elevation of ROS or AGEs might lead to a better understanding of the potential LXR-independent mechanisms in regulating $A B C A 1 / A B C G 1$ in these patients.

The impairment of $\mathrm{ABC}$ transporters might not only lead to cholesterol deposition in monocyte/macrophages to form foam cells, 
but might also relate to vascular injury in hypertensive patients. Accumulated evidence has indicated that atherogenesis is initiated by the interplay between cholesterol metabolism and cellular secretion of various cytokines. ${ }^{23}$ Cholesterol lowering might have an immune modulation in the equilibrium between sub-populations of T-helper cells ${ }^{24}$ and improve endothelial function, ${ }^{25}$ and cholesterol accumulation is considered to be associated with an inappropriate immune response to vascular injury. ${ }^{25}$ Our data suggest that monocyte cholesterol homeostasis was disturbed in our hypertensive patients because of the reduction of $\mathrm{ABC}$ transporters, which might potentially influence the function of monocytes and initiate vascular injury in these hypertensive patients.

Owing to the limited amount of blood monocytes isolated from each patient, the protein level of ABCA1, ABCG1 and SR-BI in monocytes was not measured in our study. However, we have carried out functional studies to see whether the alteration of mRNA expression in the three transporters/receptor might lead to the impairment of cholesterol efflux from monocyte-derived macrophages. We used autologous serum to induce cholesterol efflux from monocyte-derived macrophages to mimic an in vivo situation. Cholesterol efflux from macrophages to autologous serum, which mimics an early step of macrophage RCT, was significantly decreased in our hypertensive patients, and it was associated with the level of $A B C A 1$ and $A B C G 1$, but not with that of $S R-B I$. To further verify cholesterol efflux mediated by ABCA1 or ABCG1, we also used pure particles of apoAI as cholesterol acceptors to measure ABCA1-mediated cholesterol efflux. We found that cholesterol efflux from macrophages to apoAI was decreased in hypertensive patients, which was associated with the expression of $A B C A 1$, suggesting that the reduction of ABCA1 might lead to the impairment of its corresponding functional effect in cholesterol efflux to apoAI. Similarly, we also found that cholesterol efflux from macrophages to HDL was decreased in hypertensive patients, which was associated with the expression of $A B C G 1$, suggesting the reduction of $A B C G 1$ might also lead to the impairment of its corresponding functional effect in cholesterol efflux to HDL.

There are several limitations in our study. Similar to the earlier report by Zhou et al., ${ }^{9}$ we could only measure the expression of $A B C A 1, A B C G 1$ and $S R-B I$ in peripheral blood monocytes because these cells are easily accessible. However, the expression of these molecules in peripheral blood monocytes may not directly reflect the expression level in macrophages in the arterial wall. As the expression of $A B C A 1, A B C G 1$ and $S R-B I$ might be altered during the differentiation of monocytes to macrophages in vitro, ${ }^{26-28}$ mRNA was determined in freshly isolated blood monocytes for determining the basal level of gene expression in vivo. As it has been found in an earlier report that $A B C A 1 \mathrm{mRNA}$ and $\mathrm{ABCA} 1$ protein were hardly detectable in monocytes, ${ }^{29}$ in this study, we used a more sensitive method of real-time quantitative RT-PCR (reverse transcription-PCR) to detect the mRNA expression of $A B C A 1 / A B C G 1$. However, the protein levels of $\mathrm{ABC}$ transporters and SR-BI in monocytes isolated from each patient could not be determined because of the limited amount of monocytes and the hardly undetectable protein level as described by Zhou et al. ${ }^{29}$ Therefore, we have carried out functional studies to determine whether the reduction in the mRNA level was accompanied by a functional effect of the impairment of cholesterol efflux.

In summary, although hypertension is an important risk factor for the development of atherosclerosis, the underlying mechanisms are incompletely described. Our data suggest that the two important ABC transporters in the RCT pathway were reduced in hypertensive patients, which was associated with the mean blood pressure level and could be reversed by anti-hypertensive therapy. The reduction of $A B C A 1 / A B C G 1$ had functional effects of impaired cholesterol efflux from monocyte-derived macrophages; hence, the impaired RCT might at least partially contribute to the increased risk of atherosclerosis in these patients. We recruited our hypertensive patients, who were newly diagnosed as primary hypertensives during their annual examination. These findings suggested that even at the early stage of hypertension, macrophage RCT might be impaired, which might accelerate the process of atherosclerosis in these patients.

In conclusion, the expression of $A B C A 1 / A B C G 1$ in peripheral blood monocytes is reduced in hypertensive patients, which is associated with the impairment of cholesterol efflux from monocyte-derived macrophages, and may potentially contribute to accelerated foam cell formation, vascular injury and atherogenesis in these patients.

\section{CONFLICT OF INTEREST}

The authors declare no conflict of interest.

\section{ACKNOWLEDGEMENTS}

We thank the Department of Clinical Biochemistry for performing the clinical biochemical measurements and for technical assistance.

1 Lewis GF, Rader DJ. New insights into the regulation of HDL metabolism and reverse cholesterol transport. Circ Res 2005; 96: 1221-1232.

2 Jessup W, Gelissen IC, Gaus K, Kritharides L. Roles of ATP binding cassette transporters $\mathrm{A} 1$ and $\mathrm{G} 1$, scavenger receptor $\mathrm{BI}$ and membrane lipid domains in cholesterol export from macrophages. Curr Opin Lipidol 2006; 17: 247-257.

3 Van Eck M, Pennings M, Hoekstra M, Out R, Van Berkel TJ. Scavenger receptor BI and ATP-binding cassette transporter A1 in reverse cholesterol transport and atherosclerosis. Curr Opin Lipidol 2005; 16: 307-315.

4 Castelli WP, Garrison RJ, Wilson PW, Abbott RD, Kalousdian S, Kannel WB. Incidence of coronary heart disease and lipoprotein cholesterol levels. The Framingham Study. JAMA 1986; 256: 2835-2838.

5 Gordon T, Castelli WP, Hjortland MC, Kannel WB, Dawber TR. High density lipoprotein as a protective factor against coronary heart disease. The Framingham Study. Am J Med 1977; 62: 707-714.

6 Barter PJ, Rye KA. Relationship between the concentration and antiatherogenic activity of high-density lipoproteins. Curr Opin Lipidol 2006; 17: 399-403.

7 Ozerova IN, Perova NV, Shchel'tsyna NV, Mamedov MN. Parameters of high-density lipoproteins in patients with arterial hypertension incombination with other components of metabolic syndrome. Bull Exp Biol Med 2007; 143: 320-322.

8 Hasegawa K, Wakino S, Kanda T, Yoshioka K, Tatematsu S, Homma K, Takamatsu I, Sugano N, Hayashi K. Divergent action of calcium channel blockers on ATP-binding cassette protein expression. J Cardiovasc Pharmacol 2005; 46: 787-793.

9 Zhou H, Tan KC, Shiu SW, Wong Y. Determinants of leukocyte adenosine triphosphatebinding cassette transporter G1 gene expression in type 2 diabetes mellitus. Metabolism 2008; 57: 1135-1140.

10 Lei ZB, Qin YW, Shen X, Jing Q. The isolation of peripheral blood monocytes with a method using gel. Acad J Sec Mil Med Univ 1999; 20: 1039-1040 (in Chinese).

11 Gianturco SH, Ramprasad MP, Lin AH, Song R, Bradley WA. Cellular binding site and membrane binding proteins for triglyceride-rich lipoproteins in human monocytemacrophages and THP-1 monocytic cells. J Lipid Res 1994; 35: 1674-1687.

12 Wang X, Collins HL, Ranalletta M, Fuki IV, Billheimer JT, Rothblat GH, Tall AR, Rader DJ. Macrophage ABCA1 and ABCG1, but not SR-BI, promote macrophage reverse cholesterol transport in vivo. J Clin Invest 2007; 117: 2216-2224.

13 Laffitte BA, Joseph SB, Walczak R, Pei L, Wilpitz DC, Collins JL, Tontonoz P. Autoregulation of the human liver $X$ receptor alpha promoter. Mol Cell Biol 2001; 21: 7558-7568.

14 Schmitz G, Langmann T. Transcriptional regulatory networks in lipid metabolism control ABCA1 expression. Biochim Biophys Acta 2005; 1735: 1-19.

15 Ameshima S, Golpon H, Cool CD, Chan D, Vandivier RW, Gardai SJ, Wick M, Nemenoff RA, Geraci MW, Voelkel NF. Peroxisome proliferator-activated receptor gamma (PPARgamma) expression is decreased in pulmonary hypertension and affects endothelial cell growth. Circ Res 2003; 92: 1162-1169.

16 Hsueh WA, Bruemmer D. Peroxisome proliferator-activated receptor gamma: implications for cardiovascular disease. Hypertension 2004; 43: 297-305.

17 Sarafidis PA, Lasaridis AN. Actions of peroxisome proliferator-activated receptorsgamma agonists explaining a possible blood pressure-lowering effect. Am J Hypertens 2006; 19: 646-653.

18 Giles TD, Sander AG. Effects of thiazolidinediones on blood pressure. Curr Hypertens Rep 2007; 9: 332-337. 
19 Chen M, Li W, Wang N, Zhu Y, Wang X. ROS and NF-kappaB but not LXR mediate IL1 beta signaling for the downregulation of ATP-binding cassette transporter A1. Am J Physiol Cell Physiol 2007; 292: C1493-C1501.

20 Isoda K, Folco EJ, Shimizu K, Libby P. AGE-BSA decreases ABCG1 expression and reduces macrophage cholesterol efflux to HDL. Atherosclerosis 2007; 192: 298-304.

21 Puddu P, Puddu GM, Cravero E, Rosati M, Muscari A. The molecular sources of reactive oxygen species in hypertension. Blood Press 2008; 17: 70-77.

22 Baumann M, Stehouwer C, Scheijen J, Heemann U, Struijker Boudier H, Schalkwijk C. $\mathrm{N}$ epsilon-(carboxymethyl) lysine during the early development of hypertension. Ann N Y Acad Sci 2008; 1126: 201-204.

23 Kaul D. Molecular link between cholesterol, cytokines and atherosclerosis. Mol Cell Biochem 2001; 219: 65-71.

24 Hakamada-Taguchi R, Uehara Y, Kuribayashi K, Numabe A, Saito K, Negoro H, Fujita T, Toyo-oka T, Kato T. Inhibition of hydroxymethylglutaryl-coenzyme a reductase reduces Th1 development and promotes Th2 development. Circ Res 2003; 93: 948-956.
25 Vogel RA. Cholesterol lowering and endothelial function. Am J Med 1999; 107: 479-487.

26 Langmann T, Klucken J, Reil M, Liebisch G, Luciani MF, Chimini G, Kaminski WE, Schmitz G. Molecular cloning of the human ATP-binding cassette transporter 1 ( $\mathrm{hABC} 1$ ): evidence for sterol-dependent regulation in macrophages. Biochem Biophys Res Commun 1999; 257: 29-33.

27 Klucken J, Buchler C, Orso E, Kaminski WE, Porsch-Ozcurumez M, Liebisch G, Kapinsky M, Diederich W, Drobnik W, Dean M, Allikmets R, Schmitz G. ABCG1 (ABC8), the human homolog of the Drosophila white gene, is a regulator of macrophage cholesterol and phospholipid transport. Proc Natl Acad Sci USA 2000; 97: 817-822.

28 Han J, Nicholson AC, Zhou X, Feng J, Gotto Jr AM, Hajjar DP. Oxidized low density lipoprotein decreases macrophage expression of scavenger receptor B-I. J Biol Chem 2001; 276: 16567-16572.

29 Zhou X, Engel T, Goepfert C, Erren M, Assmann G, von Eckardstein A. The ATP binding cassette transporter $A 1$ contributes to the secretion of interleukin 1beta from macrophages but not from monocytes. Biochem Biophys Res Commun 2002; 291: 598-604. 